OPEN ACCESS

Edited by:

Barbara Colombo,

Champlain College, United States

Reviewed by:

Carmen María Galvez-Sánchez,

University of Jaén, Spain

Giulia Corno,

University of Quebec in Outaouais,

Canada

*Correspondence:

Loredana Cena

Ioredana.cena@unibs.it

†These authors have contributed equally to this work

Specialty section

This article was submitted to Psychology for Clinical Settings, a section of the journa

Frontiers in Psychology

Received: 09 January 2020

Accepted: 17 February 2020

Published: 11 March 2020

Citation:

Cena L, Palumbo G, Mirabella F Gigantesco A, Stefana A, Trainini A,

Tralli $N$ and Imbasciati $A$ (2020)

Perspectives on Early Screening and Prompt Intervention to Identify and Treat Maternal Perinatal Mental Health. Protocol for a Prospective

Multicenter Study in Italy.

Front. Psychol. 11:365.

doi: 10.3389/fpsyg.2020.00365

\section{Perspectives on Early Screening and Prompt Intervention to Identify and Treat Maternal Perinatal Mental Health. Protocol for a Prospective Multicenter Study in Italy}

\author{
Loredana Cena $^{1 * \dagger}$, Gabriella Palumbo ${ }^{2 \dagger}$, Fiorino Mirabella², Antonella Gigantesco², \\ Alberto Stefana ${ }^{1}$, Alice Trainini ${ }^{1}$, Nella Tralli ${ }^{1}$ and \\ Antonio Imbasciati ${ }^{1}$ for the Observatory of Perinatal Clinical Psychology Group
}

${ }^{1}$ Department of Clinical and Experimental Sciences, Section of Clinical Psychology, Observatory of Perinatal Clinical Psychology, University of Brescia, Brescia, Italy, ${ }^{2}$ Center for Behavioural Sciences and Mental Health, National Institutes of Health, Rome, Italy

Background: The most common mental disorders in women during the perinatal (antenatal and postnatal) period are depressive syndromes and anxiety syndromes. The global prevalence of maternal perinatal depression ranges from 10 to $20 \%$, while the prevalence of perinatal anxiety ranges from 10 to $24 \%$. The comorbidity of mood and anxiety disorders in perinatal women is common, reaching $40 \%$. In Italy, a few studies have been undertaken to evaluate the prevalence of perinatal depression and anxiety, and there is still a scarcity of research and intervention programs regarding primary prevention. Three of the main aims of this study are: (1) to evaluate the prevalence of maternal perinatal depression and anxiety in a large sample of women attending healthcare centers in Italy; (2) to investigate the psychosocial risks and protective factors associated with maternal perinatal depression and anxiety; (3) to evaluate the effectiveness of a manualized psychological intervention (Milgrom et al., 1999) to treat perinatal depression; (4) to evaluate the psychometric properties of both the Edinburgh Postnatal Depression Scale and the Patient Health Questionnaire-9 in detecting perinatal depression; and (5) to evaluate the influence of maternal depression and anxiety on the development of infant temperament.

Methods: This is a prospective cohort study, which merges an observational design and a pre-post intervention design. The study includes a 1-year recruitment period and a one-year follow-up period. The methodological strategy includes: (1) self-report questionnaires on maternal depression, anxiety, health status, quality of life and psychosocial risks; (2) a self-report questionnaire to measure the infant's 
temperament; (3) a clinical interview; (4) a structured diagnostic interview; and (5) a psychological intervention.

Discussion: The results of this study may contribute to our knowledge about prevalence of antenatal and postnatal depression and anxiety (during both the trimesters of pregnancy and the first six trimesters after birth) and about the effectiveness of early psychological intervention in the perinatal health services.

Keywords: perinatal, antenatal, postnatal, anxiety, depression, screening, assessment, psychological treatment

\section{INTRODUCTION}

\section{Background}

The perinatal period is a complex and vulnerable period covering the prenatal and postnatal period, which presents a series of challenges for women and men who are on their journey toward parenthood (Parfitt and Ayers, 2014). This transition can be a happy phase of life, full of positive expectations for the parents-to-be; however, it can also be a source of distress and difficulties. Indeed, it is common for pregnant women and new mothers to experience variations in mood and emotional changes due to a combination of hormonal factors and the burden of maternal responsibilities. Typically, this situation tends to resolve itself naturally, however, where there is a wide gap between expectations or resources and the reality of motherhood, psychological difficulties or mental health disorders can be consolidated or even worsen (Sipsma et al., 2016). Moreover, this situation has consequences on a larger scale because, during the perinatal period, the wellbeing and mental health of the mother, father and baby are closely interrelated (Yeaton-Massey and Herrero, 2019). Indeed, parental perinatal complications can interfere with the parentchild relationship, with the risk of significant consequences over the years (Cirulli et al., 2003; Milgrom et al., 2006; Meneghetti, 2007, 2011; Branchi et al., 2013; Stefana and Lavelli, 2017) for the child's cognitive, social and emotional development (KimCohen et al., 2005). With regards to the child, it must be taken into account that perinatal clinical psychology studies (Imbasciati et al., 2007; Imbasciati and Cena, 2015a,b) and neuroscience research (Imbasciati and Cena, 2017) show that the mother-baby-father relationships influence the construction of the child's neuropsychic structure in his brain, especially during the perinatal period (considered in our study to be the first 1000 days of life: from conception to 18 months of age) and, to an extent, throughout life (Imbasciati, 2006; Imbasciati and Cena, 2018).

The most common perinatal mental disorders in women during pregnancy and postpartum are depressive syndromes and anxiety syndromes: non-psychotic disorders characterized by the specific feelings and thoughts about the parental role (Robinson and Stewart, 2001). The global prevalence of clinically significant maternal perinatal depression ranges from 10 to $20 \%$ (Korja et al., 2018; Earls et al., 2019), while the prevalence of perinatal anxiety disorders ranges from 10 to 24\% (Dennis et al., 2018; Yeaton-Massey and Herrero, 2019). Furthermore, the comorbidity of mood disorder and anxiety disorder in perinatal women is common (Falah-Hassani et al., 2016), reaching 40\% in some studies (Reck et al., 2008; Austin et al., 2010), but often under-diagnosed (Misri and Swift, 2015). In Italy, the few studies carried out to evaluate the prevalence of perinatal depression and anxiety show large variability. Evaluations of maternal perinatal depression and anxiety record ranges of 1.626.6\% and 6.4-20.5\%, respectively (Currò et al., 2009; Mauri et al., 2010; Banti et al., 2011; Girardi et al., 2011; Giardinelli et al., 2012; Elisei et al., 2013; Palumbo et al., 2016; Clavenna et al., 2017; Di Venanzio et al., 2017; Vizzini et al., 2018). The above-mentioned data are influenced by factors such as different screening tools (which have different psychometric properties) and time periods.

Most studies in the literature undertaken by the Italian National Institute of Health and funded by the Ministry of Health - National Centre for Disease Prevention and Control (Palumbo et al., 2016, 2017, 2018) have focused mainly on the effects of maternal postnatal mental health complications. However, more recent studies (Kendig et al., 2017) have examined maternal antenatal and postnatal depression and anxiety, to understand better their short- and long-term effects on both mother and fetus/child (Dunkel Schetter and Tanner, 2012; Suri et al., 2014; Glover, 2015; van der Waerden et al., 2015; Riva Crugnola et al., 2016; Galbally and Lewis, 2017; Field, 2018; Shonkoff et al., 2019).

However, although several recent studies suggest the importance of screening early on in pregnancy in order to reduce anxiety or depressive symptoms and prevent a postpartum episode (Austin, 2004; O'Connor et al., 2016), there are still few research and intervention programs regarding primary prevention. These studies suggest the importance of identifying perinatal mental complications from the beginning of the pregnancy in order to reduce symptoms and prevent a postpartum episode through developing pathways tailored to safeguard the health of mother and child. Nevertheless, in Italy, such programs are not adequately integrated with clinical guidelines for appropriate practical care planning routines. This is due partially to the absence of an Italian policy to screen for anxiety and/or depression during the perinatal period. Additionally, there is limited training among healthcare providers on how to choose the most appropriate screening tool and the cut-off for the specific period.

Through this study, the Observatory of Perinatal Clinical Psychology (University of Brescia, Italy) and the Italian National Institute of Health aim to help promoting and improving 
the perinatal mental health prevention and early intervention programs in the Italian health service.

\section{Main Objectives}

(1) In light of the above, the first objective of this study is to evaluate the prevalence of both maternal antepartum and postpartum depression and anxiety in a large sample of women attending healthcare centers in Italy.

(2) The second objective, parallel to the first, is to investigate the psychosocial risks and protective factors associated with maternal antepartum and postpartum depression and anxiety.

(3) The third objective, building on the first, is to evaluate the psychometric properties of both the Edinburgh Postnatal Depression Scale (Cox et al., 1987) and the Patient Health Questionnaire-9 (Kroenke et al., 2001) in detecting perinatal depression.

(4) The fourth objective is to evaluate the effectiveness of a manualized psychological intervention (Milgrom et al., 1999) for both antenatal and postnatal depression in a large sample of mothers in Italy.

(5) The final objective is to evaluate the influence of maternal depression and anxiety on the development of baby temperament during a period of a minimum of 12 months up to a maximum of 18 months from birth.

\section{Hypotheses}

(1) On the basis of the current literature (Underwood et al., 2016; Okagbue et al., 2017; Hahn-Holbrook et al., 2018), we expect to find a prevalence of perinatal depression within the range of $13-18 \%$, with higher rates during pregnancy than in the first year following childbirth.

(2) Based on previous research (Ghaedrahmati et al., 2017; Leach et al., 2017), we assume that the factors associated with a higher risk of perinatal depression and anxiety will include previous history of depression and anxiety, a negative attitude toward a recent previous pregnancy, highrisk pregnancy, young maternal age, limited social support, and financial problems.

(3) In accordance with previous studies on screening for antenatal or postnatal depression (Yawn et al., 2009; Zhong et al., 2014; Santos et al., 2016), we expect that both the Edinburgh Postnatal Depression Scale and the Patient Health Questionnaire-9 will be reliable and valid scales for the assessment of perinatal depression.

(4) Based on a prior Italian study (Mirabella et al., 2016) showing the effectiveness of Jeannette Milgrom's (Milgrom et al., 1999) psychological intervention among women suffering from postpartum depression, we assume that intervention can be effective also with antepartum depressed women.

(5) Given that the literature (Erickson et al., 2017) has offered ambiguous results on the association between prenatal maternal mental health and the development of infant temperament, we have no specific assumptions to this regard.

\section{METHODS AND ANALYSIS}

\section{Study Design}

This is a prospective cohort study which merges an observational design and a pre-post intervention design. The study involves a one-year recruitment period and a one-year follow up period. The methodological strategy includes: self-report questionnaires on maternal depression, anxiety, health status, quality of life, and psychosocial risks; a self-report questionnaire to measure the infant's temperament; a clinical interview; a structured diagnostic interview; and a psychological intervention.

\section{The Observatory of Perinatal Clinical Psychology}

The Observatory of Perinatal Clinical Psychology, established at the Faculty of Medicine and Surgery of the University of Brescia (Italy) and affiliated to the Department of Clinical and Experimental Sciences, is an authorized center for perinatal studies and research, with contributions by professionals and researchers who deal with perinatal clinical psychology. The interdisciplinary collaboration network and the clinical and experimental research projects undertaken have the primary objective of protecting and promoting the mental health of women, men, couples, children and families. All this has also formed the subject of postgraduate course in Perinatal Clinical Psychology organized annually at the University of Brescia since 2012 (Scientific Directors: Imbasciati, Cena, 2012-2019) ${ }^{1}$ and attended by Italian professionals employed in Institutions responsible for providing perinatal support (e.g. clinics, hospitals, local health authorities).

\section{Reference Centre for Behavioural Sciences and Mental Health - National Institute of Health}

The Italian National Institute of Health (Istituto Superiore di Sanità, ISS) is the main Italian research institute in the field of biomedicine and public health. It is the technical and scientific body of the Italian National Health Service. Mission: Promotion and protection of national and international public health through research, surveillance, regulation, control, prevention, communication, counseling and training. Vision: The ISS produces knowledge through research and trials and disseminates scientific knowledge and evidence to decisionmakers, professional workers and citizens in order to protect and promote public health.

There is no health without mental health. This is the basis for the activity of the Reference Centre for Behavioural Sciences and Mental Health (SCIC), whose mission, in line with the 2013-2020 Mental Health Action Plan of the WHO, is to promote research and its application to improve the health of people suffering from mental disorders, their families and the general population.

\footnotetext{
${ }^{1}$ https://www.unibs.it/node/11596
} 


\section{Selection of Expert Healthcare Centers}

All participants of the past editions of the aforementioned course are contacted to gauge their center's interest in taking part in the study. Once the voluntary and free applications are collected, we proceed with the selection of those healthcare centers which meet the necessary criteria (including the presence of at least one experienced psychotherapist). The subsequent step is to send a letter to the directors of the identified centers in order to request their participation in the project. The result is that 11 centers throughout Italy take part in the study (see Table 1). In each participating healthcare center a coordinator (an experienced psychologist-psychotherapist or a child neuropsychiatrist) is identified to be responsible for set up a scientific technical committee to prepare and plan the research project adapted to their own operational reality. Coordinators are responsible for identification, recruitment, data collection, intervention and follow-up of study patients.

\section{Patient and Public Involvement}

Patients and public are not involved in the design or recruitment of other participants of this study. However patients are considered in adapting the intervention.

\section{Setting}

All mothers or pregnant women who attend one of the selected healthcare centers are invited to participate in the study. The data collection was initiated in June 2017 and will continue until November 2019.

\section{Training Program for Healthcare Professionals}

Several meetings with the participating healthcare centers take place to plan and share the logistics and the project organization with the professionals involved.

The Observatory of Perinatal Clinical Psychology, thanks to the collaboration with the Italian National Institute of Health, forms all of the healthcare professionals who are involved in the study through two specialist training courses.

The first course is 1 day of face-to-face training focused on the promotion of perinatal mental health awareness and screening. The following topics are covered: methodologies, tools, theoretical and applicative study material, to be used during the clinical practice to identify the risks of perinatal anxiety and depression. The educational objectives are: to improve knowledge of the main theories on perinatal depression and anxiety; to improve knowledge about perinatal screening procedures, to improve maternal awareness regarding perinatal depression and anxiety and to screen and evaluate the psychosocial risk factors. A further 3-day face-to-face training course is provided to the psychotherapists only, and focused on Jeannette Milgrom's psychological intervention for perinatal depression and anxiety (Milgrom et al., 1999, 2015a,b; Milgrom and Gemmil, 2015).

TABLE 1 | Healthcare Centers Involved in the Study.

\begin{tabular}{|c|c|c|c|c|}
\hline Location & Name & Unit type & $\begin{array}{l}\text { Promotion of perinatal } \\
\text { mental health awareness }\end{array}$ & $\begin{array}{l}\text { Professionals } \\
\text { involved }\end{array}$ \\
\hline Treviolo (Bergamo) & Mani di Scorta & Clinic and Family Centre & $\begin{array}{l}\text { Individual or group antenatal } \\
\text { meetings }\end{array}$ & $\begin{array}{l}\text { 1 PsyD } \\
1 \text { Midwife }\end{array}$ \\
\hline Bologna & $\begin{array}{l}\text { LHA of Bologna Child and Adolescent } \\
\text { Neuropsychiatry (Mental Health } \\
\text { Department - Pathological Addictions) }\end{array}$ & $\begin{array}{l}\text { Neuropsychiatry of Infancy and Adolescence } \\
\text { (Mental Health Department - Pathological } \\
\text { Addictions) in the NICU (Mother and Baby } \\
\text { Department) }\end{array}$ & $\begin{array}{l}\text { Individual meetings during the } \\
\text { infant's stay in the NICU }\end{array}$ & $\begin{array}{l}1 \mathrm{CN} \\
1 \text { PsyD } \\
1 \text { PsyD in training }\end{array}$ \\
\hline Brescia & Clinical Institute City of Brescia & Obstetrics and Gynecology OU & $\begin{array}{l}\text { Group antenatal meetings and } \\
\text { individual meetings postpartum }\end{array}$ & $\begin{array}{l}2 \text { PsyD } \\
1 \text { PT in training }\end{array}$ \\
\hline Enna & Umberto I Hospital & $\begin{array}{l}\text { Obstetrics and Gynecology OUC, Normal } \\
\text { Pregnancy Clinic }\end{array}$ & Individual antenatal meetings & 1 PsyD \\
\hline Milan & San Giuseppe Hospital & Obstetrics and Gynecology OU & Group antenatal meetings & 1 PsyD \\
\hline Novara & $\begin{array}{l}\text { GruppoPsychè Association, Maggiore } \\
\text { della Carità Hospital }\end{array}$ & Obstetrics and Gynecology OU & Group antenatal meetings & 3 PsyD \\
\hline Rome & Cristo Re Hospital & Obstetrics and Gynecology OUC & Group antenatal meetings & $\begin{array}{l}5 \text { PsyD } \\
1 \text { Psychologist }\end{array}$ \\
\hline Collegno (Turin) & LHA 3 of Turin & $\begin{array}{l}\text { Assistive Process, Perinatal Psychology, } \\
\text { Specialist Clinic of Perinatal Psychology, and } \\
\text { Vaccine Clinic }\end{array}$ & $\begin{array}{l}\text { Individual or group meetings } \\
\text { postpartum }\end{array}$ & $\begin{array}{l}1 \text { PsyD } \\
1 \text { Psychologist }\end{array}$ \\
\hline
\end{tabular}

$\overline{C N}=$ Child Neuropsychiatrist; $L H A=$ Local Health Authority; NICU = Neonatal Intensive Care Unit; OU = Unit/Department; OUC = Operating Unit Complex; $P$ syD $=$ Psychologist-Psychotherapist; RHS = Regional Health Service. 
Particular attention is also focused on how to conduct a clinical interview in perinatal populations. The course topics are: understanding the main elements and characteristics of the intervention program and the management of perinatal depression and anxiety; perinatal depression and anxiety assessment; clinical interviews with mothers; and application of the psychological intervention to mothers, also involving the babies and fathers.

During the study, there is regular contact between the coordinating center (the Observatory of Perinatal Clinical Psychology), the Italian National Institute of Health and the eleven participating healthcare units in Italy. Monthly Skype conference calls, Webex teleconferences, telephone consultations and periodic meetings are organized to keep all participants abreast of project progress and the stages of recruitment.

\section{Selection Criteria Inclusion Criteria}

Mothers are eligible for inclusion in the study if they meet the following criteria: (1a) they are pregnant or (1b) they have a biological newborn aged $\leq 6$ months; (2) they are able to speak and read Italian.

\section{Exclusion Criteria}

Mothers are excluded if (1) they have psychotic symptoms; (2) they exhibit non-suicidal self-harming or suicidal behavior; (3) they have issues with drug or substance abuse.

\section{Procedures}

This five-phase study comprises a perinatal mental health awareness period, a screening period (up to the baby's first vaccination), a psychodiagnostic evaluation, an intervention period (10 weeks' duration), and a post-intervention follow-up (up to 12 months from the end of intervention). The timeline of the whole procedure is depicted in Figure 1.

\section{Phase 1: Promoting Perinatal Mental Health Awareness}

All mothers assisted in the healthcare centers are informed about the perinatal mental health problems that can occur during pregnancy and postpartum, and their impact on both the mother's health and the baby's development. Subsequently, mothers are invited to participate in the perinatal depression screening.

\section{Phase 2: Screening for Psychosocial Risks, Depression, and Anxiety}

Screening is performed once during the pre- or post-partum period, depending on the characteristics of each healthcare center. All mothers complete the Psychosocial and Clinical Assessment Form (Mirabella et al., 2016), the Italian versions of both the Edinburgh Postnatal Depression Scale (EPDS) (Cox et al., 1987; Benvenuti et al., 1999) and the Patient Health Questionnaire-9 (PHQ-9) (Kroenke et al., 2001) to evaluate depressive symptoms and the Italian version of the State-Trait Anxiety Inventory (STAI) (Spielberger et al., 1983; Pedrabissi and Santinello, 1992) to evaluate anxiety.
Mothers are required to complete the psychodiagnostic evaluation within one week of the date of positive screening results.

\section{Phase 3: Psychodiagnostic Evaluation}

All mothers undergo an interview with a clinical psychologist. Within the following week, in the case of clinician-generated diagnosis of perinatal depression and/or anxiety (according to the DSM-5 criteria), these mothers are further assessed using the Mini-International Neuropsychiatric Interview (MINI) Plus (Lecrubier et al., 1997; Sheehan et al., 1998) to confirm the diagnosis, the World Health Organization Quality of Life (WHOQOL) BREF (The Whoqol Group, 1998) to assess the quality of life and the Italian Questionnaires of Temperament (QUITs) (Axia, 2002) to assess the babies' traits in temperament.

All mothers receive verbal feedback about the results of the psychodiagnostic evaluation. Depressed and/or anxious mothers are invited to participate in the intervention phase of the study. Whereas mothers with postpartum psychotic disorders, nonsuicidal self-harm tendencies or suicidal ideation are sent to a psychiatric service and excluded from the study.

\section{Phase 4: Intervention}

The intervention is based on the model developed by Milgrom at the Australian Parent-Infant Research Institute (PIRI) at Heidelberg Repatriation Hospital (Milgrom et al., 1999, 2011, 2015a,b; Milgrom and Gemmil, 2015) to reduce maternal prenatal and postnatal depression, anxiety and parenting difficulties. Moreover, this model provides for the simultaneous assessment of the neurobiological, psychological and social factors that contribute to developing psychological vulnerability in the perinatal period.

Milgrom developed a psychosocial and psychological treatment by adapting Cognitive-Behavioral Therapy (CBT) interventions for women suffering from depression both in pregnancy and postpartum (Milgrom et al., 1999; Milgrom and Gemmil, 2015). The effectiveness of this treatment has been assessed in both short- and long-term application, and its validity has been demonstrated and compared to pharmacological therapy using antidepressants. It has been deemed more effective than drugs in maintaining long-term results (Milgrom et al., 2015a).

The intervention consists of group-based CBT and focuses on the women's life events and mood and on practical issues. The intervention takes place primarily in groups because the group setting allows participants to meet, compare and share their experiences, offering mutual support. However, in the event of impossibility of organizing a group of at least three participants within a few weeks of the psychodiagnostic evaluation, or where a participant is unable to take part regularly in weekly group sessions due to health or organizational reasons, each healthcare center offer the option of individual treatment. This solution allows a more flexible approach than group treatment, since the program can be adapted to the specific situation of the woman in question. In both cases, information and activity material are distributed between sessions. 


\section{Promotion of mental health awareness}

Screening
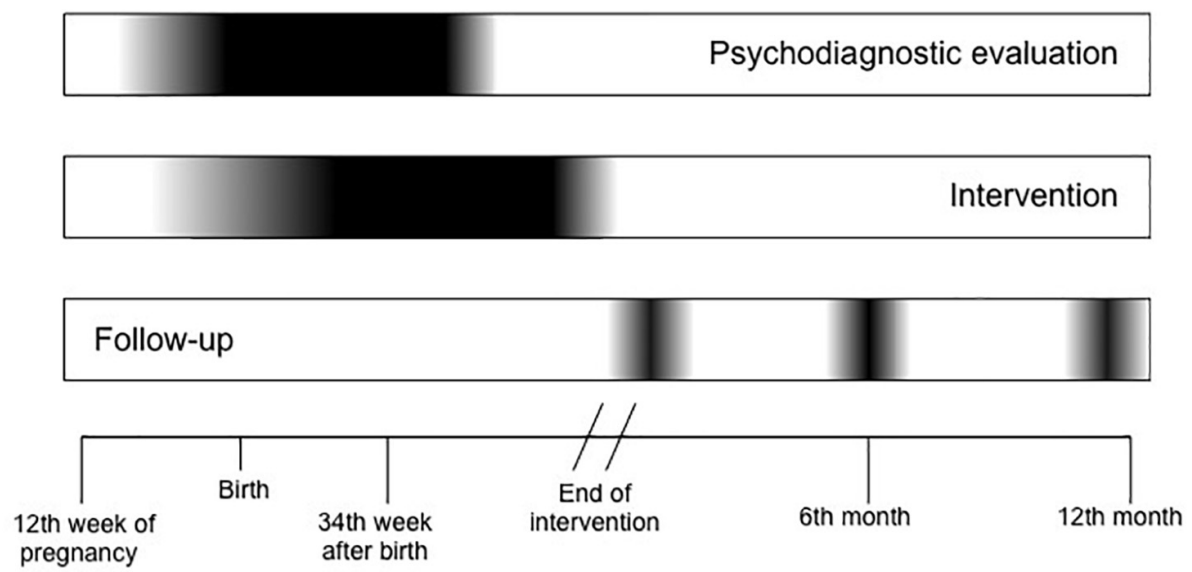

FIGURE 1 | Timeline.

This clinic-based group intervention is led and facilitated by a licensed psychotherapist and consists of ten weekly sessions of 90 min exclusively dedicated to the pregnant women/new mothers, three sessions involving the partners/fathers, and another three sessions dedicated to mother-child interaction. [In our study, before this last module, the QUITs (Axia and Weisner, 2002) is administered to assess infant temperament traits]. The content of the individual counseling is the same as that of group-based counseling and consists of ten sessions of $60 \mathrm{~min}$. At the end of each session, participants receive information and activity materials to be used in the next session. Between sessions, participants are encouraged to put what they have learnt/experienced during the treatment into practice in everyday life. The session program is shown in Table 2 .

In Italy, following the publication of the Italian language manual (Milgrom, 2003), the actual feasibility and effectiveness of Milgrom's psychological interventions for the prevention and treatment of postpartum depression has been demonstrated by ISS research (Mirabella et al., 2016).

\section{Phase 5: Follow-up}

Changes in the patients' clinical conditions are re-evaluated at three points of follow-up (end of intervention, 6th month and 12th month) using EPDS, PHQ-9, STAI, WHOQOL-BREF, QUITs, MINI-Plus and a clinical interview.

Mothers who are still depressed after the intervention phase are sent to a clinical psychological or psychiatric service.

\section{Study Outcomes}

The data on maternal psychosocial and clinical conditions will be gathered. The primary outcomes are maternal depressive and anxiety symptoms severity. Secondary outcomes are maternal perceived quality of life and infants' temperament (only for the infants whose mothers participated in the intervention).

\section{Measures}

\section{Edinburgh Postnatal Depression Scale}

The EPDS (Cox et al., 1987) is the most widely used screening tool for depressive symptoms during the perinatal period (de Moraes et al., 2017; Ukatu et al., 2018). It is a self-rating scale containing 10 items about symptoms of depression such as anhedonia, feelings of guilt, lethargy, sleep disturbance and suicidal ideation. The answer to each item is on a four-point Likert scale with which mothers indicate the frequency with which they have experienced the corresponding symptom during the past 7 days. The total score ranges from 0 to 30 , with higher scores indicating more severe depressive symptoms. The internal consistency of the Italian version of the EPDS (Cronbach standardized alpha) was 0.80 (Benvenuti et al., 1999), which is similar to the value reported by Cox in the first validation study (0.87). The sensitivity, specificity, and positive predictive value of the Italian version were recorded as 55.6, 98.9, and 90.9\%, respectively, based on a cut-off score $\geq 12$, making it a reliable and valid screening tool for perinatal depression. Furthermore, a recent study (Smith-Nielsen et al., 2018) found that a score of 11 or more is the optimal cut-off for depression according to both DSM-5 (American Psychiatric Association, 2013) and ICD-10 criteria (World Health Organization, 1992).

\section{Patient Health Questionnaire-9}

The PHQ-9 (Kroenke et al., 2001) is the first choice for depressive symptoms in non-psychiatric primary care settings 
(Thase, 2016) and the third most common screening tool for postpartum depression (de Moraes et al., 2017). It is a self-rating scale containing nine items about symptoms of depression such as anhedonia and low mood, in conjunction with problems concerning physical activity, appetite, concentration, energy, self-esteem, sleep and suicidal ideation. The answer to each item is on a four-point Likert scale with which mothers indicate the frequency with which they have experienced the corresponding symptom during the past 14 days. The total score ranges from 0 to 27 , with higher scores indicating more severe depressive symptoms. During the perinatal period, a score of $\geq 10$ is usually recommended to define the presence of depression (Flynn et al., 2011; Sidebottom et al., 2012; Marcos-Nájera et al., 2018). Furthermore, two of these

TABLE 2 | Treatment Program.

\section{Step 1: Behavioral interventions}

Session $1 \quad$ Understanding and dealing with depression.

The sessions involve the individual or group development of the themes of the treatment program. The first session begins with participant and the therapist introducing themselves, sharing experiences to encourage a sense of belonging within the group. This psychoeducational session invites women describe their expectations of motherhood and the therapist provides information on prenatal and postnatal depression.

Session 2 Pleasurable activities: where do I find the time?

The therapist encourages the women to think about their daily routine and to talk about their difficulties, thoughts, emotions and joys. Possible pleasurable activities (to enjoy alone, with their child and with their partner) are suggested for reducing the stress and fatigue of everyday commitments.

Session 3 Quick relaxation strategies.

The therapist proposes some relaxation techniques (for example, Jacobson's progressive muscle relaxation). Together with participants, the therapist examines possible simple "stress-eliminating" techniques.

Session $4 \quad$ Assertiveness and self-esteem: telling others what I think and how I feel.

The therapist proposes role-playing and communication techniques intended to encourage the women to express their needs in an assertive manner.

\section{Step 2: Cognitive interventions}

Session $5 \quad$ Unrealistic expectations on childcare: how the past can influence the present.

This session reflects on the image of motherhood and the mother. Childcare methods learned from the family of the mother-to-be are compared with those adopted in the partner's family.

Session $6 \quad$ My inner voice: the missing link.

The therapist helps participants to understand the impact that thoughts, emotions and behaviors can have on mothers' everyday life and proposes new ways to deal with negative thoughts, cognitive distortions and/or reasoning errors, by replacing them with more functional thoughts for their well-being.

Session $7 \quad$ Developing a more effective way of thinking.

In this and in subsequent sessions, the therapist helps participants to focus on techniques to encourage positive thoughts.

Session $8 \quad$ Query/challenge your inner critic.

Questioning irrational beliefs and negative/critical thoughts.

Step 3: Prevention of relapse

Session $9 \quad$ Putting it all together: "moving forward".

Mothers are encouraged to build and use new social networks so that they can refer to them for social support when treatment is concluded.

Session $10 \quad$ Consolidating what I have learned.

Session to reinforce treatment education and experiences. Mothers can bring their babies.

\section{Additional clinical forms with fathers}

Session $1 \quad$ Involving fathers: fathers and post-natal depression.

In subsequent sessions, fathers are also involved. The therapist provides fathers with information on perinatal depression. This particular session encourages couples to express their feelings about being parents and how they have felt in the past few weeks.

Session 2 Couple relationship.

This session addresses the changes that have occurred in the couple's relationship since the baby's birth. Couples are encouraged to communicate and spend quality time together.

Session 3 Do it on your own.

Summarizing the treatment experience and looking at how each couple is dealing with issues related to planning family life with the child.

Additional clinical forms with the presence of children: baby H.U.G.S

Session $1 \quad$ Let's play! Games and physical contact.

The therapist introduces neonatal massage technique and talks about the feelings generated by of physical contact. Parents are presented with games for different ages and emphasis is given to the pleasure of proper mother-child interaction and the importance of non-verbal communication.

Session 2 Let's learn something about our child. Observing and understanding his/her signals.

Activities involving non-verbal interactive behavior are recommended, including stress responses. Parents are encouraged to observe and interpret the child's non-verbal communication in a playful and fun context. The therapist introduces stimulation activities to identify differences in temperament.

Session 3

Let's analyze our feelings. Parental responses to the signals of their newborn.

Couples are encouraged to observe the differences between their needs and those of their newborn baby. 
studies evaluated the factor structure of the PHQ-9, finding that a three-factor (cognitive-affective, somatic, pregnancyrelated) model is adequate during pregnancy (Marcos-Nájera et al., 2018). The internal reliability (Cronbach alpha) of the PHQ-9 was 0.86 in an obstetrics-gynecology sample (Kroenke et al., 2001). The sensitivity, specificity, and positive predictive value of the PHQ-9 Italian version (Mazzotti et al., 2003) were recorded as 39, 29, and 93\%, respectively, for any depressive syndrome. Furthermore, the PHQ-9 can be used to assess depression severity based on the DSM-5 (American Psychiatric Association, 2013) diagnostic criteria (Spitzer et al., 2014).

\section{State-Trait Anxiety Inventory}

The STAI (Spielberger et al., 1983) is the most widely used screening tool to identify anxiety in pregnancy (Brunton et al., 2015). It is a self-rating scale containing 40 items divided into two subscales of 20 items each, which evaluate different types of anxiety: the state subscale measures anxiety in the current situation or time period, while the trait subscale measures relatively stable individual aspects of propensity toward elevated anxiety. The answer to each item is on a four-point Likert scale. The total score ranges from 20 to 80 , with higher scores indicating more severe anxiety. The STAI internal consistency ranged from 0.86 to 0.95 (Spielberger et al., 1983; Spielberger, 1989) and the Italian version (Pedrabissi and Santinello, 1992) showed psychometric properties consistent with the English version. The adopted cut-off score was $\geq 40$, as suggested by the STAI Italian version (Pedrabissi and Santinello, 1992), validated on a non-pregnant population. The construct and content validity of the STAI for pregnant woman has been proven (Gunning et al., 2010). The sensitivity, specificity, and positive predictive value of the STAI for the third trimester of pregnancy were recorded as 80.95, 79.75, and 51.50\%, respectively, based on a cut-off score of $>40$ for both state and trait scales (Grant et al., 2008). A cut-off score of $>40$ for the postpartum period is recommended (Dennis et al., 2013). The state scale of the STAI is strongly correlated with the EPDS (Meades and Ayers, 2011).

\section{Psychosocial and Clinical Assessment Form}

The Psychosocial and Clinical Assessment Form (Palumbo et al., 2016) is developed by the Italian National Institute of Health. There are two questionnaires: a pregnancy and a postnatal version. The pregnancy version is composed of 22 items addressing sociodemographic characteristics, information on mental health before pregnancy, stressful life events experienced over the last twelve months, information about the pregnancy and currently perceived family and social support. Whereas the postnatal version has an additional seven items to gather information on childbirth. The resulting psychosocial and clinical risks and protective factors can be weighted and summarized into two different psychological constructs: (a) perceived confidence in personal abilities and own social resources, and (b) perceived psychological vulnerability and ability to cope with the difficulties.

\section{Italian Questionnaires of Temperament}

The QUIT (Axia, 2002) is a set of questionnaires that are used to measure temperament in infants from the first month up to 11 years of age. This study uses the QUIT 1-12 and 12-36 month Scales. It is a caregiver-report questionnaire validated with reference to Italian culture, containing 55 items investigating six dimensions: attention, inhibition to novelty, motor activities, positive emotionality, negative emotionality and social orientation. The first three dimensions relate to adaptation in general, while the second three relate to specific adaptation to the social world. The answer to each item is on a six-point Likert scale with which mothers indicate their baby's positive or negative attitude toward the environment, with higher scores indicating greater intensity of a characteristic. Four infant temperamental profiles can be obtained on the basis of the orthogonal dimensions of positive and negative emotionality: emotional temperament, calm temperament, normal temperament, difficult temperament. The QUIT has acceptable internal consistency with Cronbach's alpha ranging from 0.59 to 0.71 (Axia, 2002), and the Pearson's correlation coefficient among reports by mothers and fathers ranges from 0.32 to 0.62 (Axia and Weisner, 2002).

\section{World Health Organization Quality of Life BREF}

The WHOQOL-BRIEF (The Whoqol Group, 1998) is a generic instrument to assess perceived quality of life (QoL). It is a self-rating questionnaire containing 28 items, of which 26 are used to measure four domains related to QoL: physical health, psychological, social relationships and environment. A further two items are used to measure individual perceptions of overall QoL and general health. The answer to each item is on a fivepoint Likert scale with which mothers indicate their degree of satisfaction/dissatisfaction. Domain scores are calculated by multiplying the mean score of all items within each domain by four and then they are transformed into weighted scores from 0 to 100 , with higher scores indicating greater levels of satisfaction. There is no an overall score. The WHOQOL-BRIEF Italian version showed good internal consistency, with Cronbach's alpha ranging from 0.65 for the social relationships domain to 0.80 for the physical domain (de Girolamo et al., 2000). The WHOQOLBREF is widely used to measure the QoL of women during the perinatal period (Webster et al., 2010; Nascimento et al., 2011; Mortazavi et al., 2014; Moura et al., 2017).

\section{Mini-International Neuropsychiatric Interview-Plus}

The MINI-Plus (Sheehan et al., 1998) is a short, structured and standardized diagnostic interview developed to assess the diagnosis of psychotic and mood disorders based on DSM-IV criteria. Its sensitivity, specificity, and reliability are equivalent to those of the Structured Clinical Interview for DSM-IV in different settings (psychiatric and primary care centers) (Sheehan et al., 1998; Amorim, 2000). This study uses the module for depressive disorders, which addresses the frequency, intensity, and duration of depressive symptoms and the degree of the associated distress and impairment. For major depressive disorders, the interrater reliability of the MINI Italian version was 0.96 (Rossi et al., 2004), indicating "excellent agreement." 


\section{Sample Size}

Assuming $10-15 \%$ prevalence of perinatal depression as measured by EPDS (Gavin et al., 2005; Melville et al., 2010) the minimum number needed of mothers to be screened is 500 .

With regard to the clinical trial on the psychological interventions for perinatal depression and anxiety, the power calculation was based on detecting an effect size of medium magnitude $(d=0.55)$ at the follow-ups on the EPDS among the patients (Cohen, 1988). We calculated that a sample size of 45 patients would result in a power of 0.95 with alpha set at 0.05 . To protect against an anticipated loss to follow-up of about $30 \%$ (Mirabella et al., 2016), we planned to enroll 65 patients.

\section{Quality Control}

The healthcare centers enter all the data collected with the instruments during the Assessment into an Excel database, prepared by the ISS (baseline + follow-up). Once the centers have entered all the data collected, each researcher (coordinator of the healthcare centers) performs a detailed quality control assessment. After this assessment by each center, the data is sent to the Observatory of Perinatal Clinical Psychology (coordinating center), which repeats the same checks, to ensure that the final database contain no errors and have no missing data.

\section{Data Analysis Plan}

The prevalence of antenatal and postpartum depression and the risk of anxiety will be calculated and compared. Since previous studies have ascertained a higher risk of developing depression in the postpartum period, the antenatal period will be taken as a reference to calculate the odds ratio as a measure of the relative risk of depression between antenatal and postpartum periods, using chi-square as a test for statistical significance.

The sensitivity, specificity and predictive values of the EPDS and the PHQ-9 versus the MINI will be analyzed. Factors associated with ante- and post-partum depression and anxiety risk will be analyzed using a logistic regression model.

With regard to the effectiveness of treatment, standard descriptive statistics will be calculated for all questionnaires. Analysis of variance for repeated measurements will be performed to compare the differences between each initial score obtained with the different administered questionnaires and the scores at T1 (end of treatment), T2 (after 6 months) and T3 (after 12 months) will be evaluated. Bonferroni post hoc comparisons will be conducted. Treatment effect will be calculated using Cohen's d for each of the follow-up times. The Reliable Change Index will be calculated for each time, in order to determine any significant clinical changes occurring in each individual case post treatment.

Data will be analyzed using the Statistical Package for Social Science (SPSS) for Windows version 22.0.

\section{ETHICS AND DISSEMINATION}

\section{Description of Risks}

There are no risks associated with the participation in any aspect of the described study.

\section{Informed Consent}

Before taking part in the study (which was approved by the Ethical Committee of the Healthcare Centre of Bologna Hospital), all subjects receive information orally and in writing about the content and implications of the study. If they are willing to participate, they have to sign the informed consent form. Recruited subjects are able to withdraw from the study at any time without explanation and with no consequences regarding the care they are receiving.

\section{Data Protection}

All transfers of study data are informed by and undertaken according to the European Parliament and the Council of Europe's Directive 95/46/EC on the protection of individuals with reference to the processing of personal data. To ensure the security and integrity of data, an appropriate documented standard procedure is established and followed without exception. Any study data that is to be transferred from research sites to the coordinating site are anonymized prior to transfer: each subject is identified with an ID code composed of five alphanumeric characters. All collected data (questionnaires, sheets with test/scale answers, documents, etc.) are marked with the ID code and do not contain any identifying information. Using this ID code, data are entered into the database. Electronic data are stored on a password protected computer and paper material is stored in the locked archive of each healthcare center. Only members of the study team have access to the data. After entering the data into the electronic database and checking data integrity, all paperwork is stored in accordance with the Italian law on privacy (GDPR, May 25, 2018). Personal information about potential and enrolled participants is collected, shared and stored in a manner that protects their confidentiality before, during and after the project.

\section{Scientific, Clinical, and Social Impact}

The project will enable the application of a perinatal depression and anxiety screening procedure that can be developed for the use in different structures, as it requires the collaboration and connection between existing structural and functional resources. The project also recommends the application of early psychological intervention in the health services to prevent perinatal depression and anxiety complications in mothers and also recommends the involvement of fathers and babies. It should be noted that underestimating these disorders could have very negative effects on public health. Early intervention on perinatal depression and anxiety can reduce the direct and indirect costs of damage to the mother in terms of personal, social and working life, and, above all, reduce the direct and indirect costs that may arise due to the impact on child development. For the individual subjects of the study, the expected benefits are the more rapid identification and treatment of anxiety and depressive disorders or other psychiatric disorders, where present, with the consequent possible improvement of mental health outcomes and greater psychological well-being.

Future research should focus on identifying the risk and protective factors with regard to the child's mental 
health. Furthermore, due to the principle of transgenerational inheritance (Imbasciati et al., 2011) it is also important to conduct prospective longitudinal studies in order to identify the effects on children who become adults and the consequent possible difficulties they might encounter in their relationship with their own children.

\section{Strengths and Limitations of This Study}

This study presents some strengths and limitations that should be mentioned. The main strengths are: (a) this study provides a new knowledge about the prevalence of perinatal depression and anxiety during both the trimesters of pregnancy and the first four trimesters after birth; (b) the large sample size is based on eleven recruitment centers located throughout Italy, and then it is representative of the pregnant population in Italy; and (c) all mothers were assessed for depression and anxiety through both self-report questionnaires and clinical interviews. The main limitation of the study is that it cannot monitor the timing and patterns of onset, duration, remission and recurrence of anxiety and depression during the entire perinatal period. Another limitation is that this protocol is the absence of a control group or waiting list condition.

\section{ETHICS STATEMENT}

The study protocol is approved by the Ethical Committee of the Healthcare Center of Bologna Hospital (Register Number: 0077805, June 27, 2017). The participants provided their written informed consent to participate in this study.

\section{REFERENCES}

American Psychiatric Association (2013). Diagnostic and Statistical Manual of Mental Disorders (DSM-5 $\left.{ }^{\circledR}\right)$. Washington, DC: American Psychiatric Association.

Amorim, P. (2000). Mini international neuropsychiatric interview (MINI): validação de entrevista breve para diagnóstico de transtornos mentais. Rev. Bras. Psiquiatr. 22, 106-115. doi: 10.1590/S1516-44462000000300003

Austin, M. P. (2004). Antenatal screening and early intervention for "perinatal" distress, depression and anxiety: where to from here? Arch. Womens. Ment. Health 7, 1-6. doi: 10.1007/s00737-003-0034-4

Austin, M. P. V., Hadzi-Pavlovic, D., Priest, S. R., Reilly, N., Wilhelm, K., Saint, K., et al. (2010). Depressive and anxiety disorders in the postpartum period: how prevalent are they and can we improve their detection? Arch. Womens. Ment. Health 13, 395-401. doi: 10.1007/s00737-0100153-7

Axia, G. (2002). QUIT: Questionari Italiani del Temperamento. Gardolo: Erikson.

Axia, V. D., and Weisner, T. S. (2002). Infant stress reactivity and home cultural ecology of Italian infants and families. Infant Behav. Dev. 25, 255-268. doi: 10.1016/S0163-6383(02)000991

Banti, S., Mauri, M., Oppo, A., Borri, C., Rambelli, C., Ramacciotti, D., et al. (2011). From the third month of pregnancy to 1 year postpartum. Prevalence, incidence, recurrence, and new onset of depression. Results from the perinatal depression-research \& screening unit study. Compr. Psychiatry 52, 343-351. doi: 10.1016/j.comppsych.2010.08.003

Benvenuti, P., Ferrara, M., Niccolai, C., Valoriani, V., and Cox, J. L. (1999). The edinburgh postnatal depression scale: validation for an Italian sample. J. Affect. Disord. 53, 137-141. doi: 10.1016/S0165-0327(98)00102-5

Branchi, I., Curley, J. P., D’Andrea, I., Cirulli, F., Champagne, F. A., and Alleva, E. (2013). Early interactions with mother and peers independently

\section{AUTHOR CONTRIBUTIONS}

This study design is developed in mutual agreement of scientific collaboration between the Observatory of Perinatal Clinical Psychology and the Italian National Institute of Health. LC, GP, and AI contributed equally to the general study design. FM designed the plan of statistical analysis of the study protocol. AG serves primarily as research statistical analysis supervisor. LC and AT from the Observatory coordinate and manage the implementation of the Study in each Healthcare Center. LC, AI, AS, and AT contributed to preparing the manuscript. All authors agreed to submit the final version of the manuscript.

\section{FUNDING}

This work was self-funded by the Observatory of Perinatal Clinical Psychology - Department of Clinical and Experimental Sciences of the University of Brescia (Italy).

\section{ACKNOWLEDGMENTS}

We would like to thank all the mothers, babies, and fathers who take part in the study in each of the Healthcare Centers in Italy. We also would like to thank all the professionals who participate in the execution of this study. This manuscript has been released as a pre-print at ISRCTN registry (Cena, 2019) doi: 10.1186/ISRCTN12733828.

build adult social skills and shape BDNF and oxytocin receptor brain levels. Psychoneuroendocrinology 38, 522-532. doi: 10.1016/j.psyneuen.2012.07.010

Brunton, R. J., Dryer, R., Saliba, A., and Kohlhoff, J. (2015). Pregnancy anxiety: a systematic review of current scales. J. Affect. Disord. 176, 24-34. doi: 10.1016/j. jad.2015.01.039

Cena (2019). Early screening and prompt intervention to identify and treat maternal mental health problems before and after giving birth. BMC Res. Prog. [Preprint]. doi: 10.1186/ISRCTN12733828

Cirulli, F., Berry, A., and Alleva, E. (2003). Early disruption of the mother-infant relationship: effects on brain plasticity and implications for psychopathology. Neurosci. Biobehav. Rev. 27, 73-82. doi: 10.1016/S0149-7634(03) 00010-1

Clavenna, A., Seletti, E., Cartabia, M., Didoni, A., Fortinguerra, F., Sciascia, T., et al. (2017). Postnatal depression screening in a paediatric primary care setting in Italy. BMC Psychiatry 17:42. doi: 10.1186/s12888-017-1205-6

Cohen, J. (1988). Statistical Power Analysis for the Behavioral Science. Hillsdale, NJ: Lawrence Erlbaum Associates.

Cox, J. L., Holden, J. M., and Sagovsky, R. (1987). Detection of postnatal depression: development of the 10-item edinburgh postnatal depression scale. Br. J. Psychiatry 150, 782-786. doi: 10.1192/bjp.150.6.782

Currò, V., De Rosa, E., Maulucci, S., Maulucci, M., Silvestri, M., Zambrano, A., et al. (2009). The use of edinburgh postnatal depression scale to identify postnatal depression symptoms at well child visit. Ital. J. Pediatr. 35:32. doi: 10.1186/18247288-35-32

de Girolamo, G., Rucci, P., Scocco, P., Becchi, A., Coppa, F., D’Addario, A., et al. (2000). Quality of life assessment: validation of the Italian version of the WHOQOL-Brief. Epidemiol. Psichiatr. Soc. 9, 45-55. doi: 10.1017/ S1121189X00007740

de Moraes, G. P. A., Lorenzo, L., Pontes, G. A. R., Montenegro, M. C., and Cantilino, A. (2017). Screening and diagnosing postpartum depression: when 
and how? Trends Psychiatry Psychother. 39, 54-61. doi: 10.1590/2237-60892016-0034

Dennis, C.-L., Coghlan, M., and Vigod, S. (2013). Can we identify mothers at-risk for postpartum anxiety in the immediate postpartum period using the StateTrait Anxiety Inventory? J. Affect. Disord. 150, 1217-1220. doi: 10.1016/j.jad. 2013.05.049

Dennis, C.-L., Falah-Hassani, K., and Shiri, R. (2018). Prevalence of antenatal and postnatal anxiety: systematic review and meta-analysis. Br. J. Psychiatry 210, 315-323. doi: 10.1192/bjp.bp.116.187179

Di Venanzio, C., Pacitti, F., Rossetti, M. C., Santarelli, V., Gregori, E., D’Alfonso, A., et al. (2017). Perinatal depression screening and early treatment. J. Psychopathol. 23, 99-104.

Dunkel Schetter, C., and Tanner, L. (2012). Anxiety, depression and stress in pregnancy. Curr. Opin. Psychiatry 25, 141-148. doi: 10.1097/YCO. 0b013e3283503680

Earls, M. F., Yogman, M. W., and Mattson, G. (2019). AAP committee on psychosocial aspects of child and family health. incorporating recognition and management of perinatal depression into pediatric practice. Pediatrics 143:20183259HQ.

Elisei, S., Lucarini, E., Murgia, N., Ferranti, L., and Attademo, L. (2013). Perinatal depression: a study of prevalence and of risk and protective factors. Psychiatr. Danub. 25(Suppl. 2), S258-S262.

Erickson, N. L., Gartstein, M. A., and Dotson, J. A. W. (2017). Review of prenatal maternal mental health and the development of infant temperament. J. Obst. Gynecol. Neonatal Nurs. 46, 588-600. doi: 10.1016/j.jogn.2017.03.008

Falah-Hassani, K., Shiri, R., and Dennis, C.-L. (2016). Prevalence and risk factors for comorbid postpartum depressive symptomatology and anxiety. J. Affect. Disord. 198, 142-147. doi: 10.1016/j.jad.2016.03.010

Field, T. (2018). Postnatal anxiety prevalence, predictors and effects on development: a narrative review. Infant Behav. Dev. 51, 24-32. doi: 10.1016/j. infbeh.2018.02.005

Flynn, H. A., Sexton, M., Ratliff, S., Porter, K., and Zivin, K. (2011). Comparative performance of the edinburgh postnatal depression scale and the patient health questionnaire-9 in pregnant and postpartum women seeking psychiatric services. Psychiatry Res. 187, 130-134. doi: 10.1016/j.psychres.2010.10.022

Galbally, M., and Lewis, A. J. (2017). Depression and parenting: the need for improved intervention models. Curr. Opin. Psychol. 15, 61-65. doi: 10.1016/j. copsyc. 2017.02 .008

Gavin, N. I., Gaynes, B. N., Lohr, K. N., Meltzer-Brody, S., Gartlehner, G., and Swinson, T. (2005). Perinatal depression. Obstet. Gynecol. 106, 1071-1083. doi: 10.1097/01.AOG.0000183597.31630.db

Ghaedrahmati, M., Kazemi, A., Kheirabadi, G., Ebrahimi, A., and Bahrami, M. (2017). Postpartum depression risk factors: a narrative review. J. Educ. Health Promot. 6:60. doi: 10.4103/jehp.jehp_9_16

Giardinelli, L., Innocenti, A., Benni, L., Stefanini, M. C., Lino, G., Lunardi, C., et al. (2012). Depression and anxiety in perinatal period: prevalence and risk factors in an Italian sample. Arch. Womens Ment. Health 15, 21-30. doi: 10. 1007/s00737-011-0249-8

Girardi, P., Pompili, M., Innamorati, M., Serafini, G., Berrettoni, C., Angeletti, G., et al. (2011). Temperament, post-partum depression, hopelessness, and suicide risk among women soon after delivering. Women Health 51, 511-524. doi: $10.1080 / 03630242.2011 .583980$

Glover, V. (2015). Prenatal stress and its effects on the fetus and suicide risk among women soon after delivering. Perinat. Program. Neurodev. 10, 269-283. doi: 10.1007/978-1-4939-1372-5_13

Grant, K.-A., McMahon, C., and Austin, M.-P. (2008). Maternal anxiety during the transition to parenthood: a prospective study. J. Affect. Disord. 108, 101-111. doi: 10.1016/j.jad.2007.10.002

Gunning, M. D., Denison, F. C., Stockley, C. J., Ho, S. P., Sandhu, H. K., and Reynolds, R. M. (2010). Assessing maternal anxiety in pregnancy with the StateTrait Anxiety Inventory (STAI): issues of validity, location and participation. J. Reprod. Infant Psychol. 28, 266-273. doi: 10.1080/0264683090348 7300

Hahn-Holbrook, J., Cornwell-Hinrichs, T., and Anaya, I. (2018). Economic and health predictors of national postpartum depression prevalence: a systematic review, meta-analysis, and meta-regression of 291 studies from 56 countries. Front. Psychiatry 8:248. doi: 10.3389/fpsyt.2017.00248
Imbasciati, A. (2006). Constructing a Mind: A New Base for Psychoanalytic Theory. London: Routledge.

Imbasciati, A., and Cena, L. (2015a). Psicologia Clinica Perinatale per le Professioni Sanitarie E Psicosociali. Vol. I. Neonato e Radici Della Salute Mentale. Milano: Franco Angeli.

Imbasciati, A., and Cena, L. (2015b). Psicologia Clinica Perinatale per le Professioni Sanitarie e Psicosociali. Vol. 2: Genitorialità e Origini Della Mente del Bambino. Milano: FrancoAngeli.

Imbasciati, A., and Cena, L. (2017). Psicologia Clinica Perinatale - Neuroscienze e Psicoanalisi. Milano: Franco Angeli.

Imbasciati, A., and Cena, L. (2018). Il futuro dei Primi Mille Giorni di Vita. Milano: Franco Angeli.

Imbasciati, A., Dabrassi, F., and Cena, L. (2007). Psicologia Clinica Perinatale. Padova: Piccin.

Imbasciati, A., Dabrassi, F., and Cena, L. (2011). Psicologia Clinica Perinatale per il Futuro Individuo: Un Uomo Transgenerazionale. Torino: Ed Express.

Kendig, S., Keats, J. P., Hoffman, M. C., Kay, L. B., Miller, E. S., Moore Simas, T. A., et al. (2017). Consensus bundle on maternal mental health: perinatal depression and anxiety. J. Obstet. Gynecol. Neonatal Nurs. 46, 272-281. doi: 10.1016/j.jogn.2017.01.001

Kim-Cohen, J., Moffitt, T. E., Taylor, A., Pawlby, S. J., and Caspi, A. (2005). Maternal depression and children's antisocial behavior. Arch. Gen. Psychiatry 62, 173-181. doi: 10.1001/archpsyc.62.2.173

Korja, R., Nolvi, S., Kataja, E.-L., Scheinin, N., Junttila, N., Lahtinen, H., et al. (2018). The courses of maternal and paternal depressive and anxiety symptoms during the prenatal period in the FinnBrain Birth Cohort study. PLoS One 13:e0207856. doi: 10.1371/journal.pone.0207856

Kroenke, K., Spitzer, R. L., and Williams, J. B. W. (2001). The PHQ-9: validity of a brief depression severity measure. J. Gen. Intern. Med. 16, 606-613. doi: 10.1046/j.1525-1497.2001.016009606.x

Leach, L., Poyser, C., and Fairweather-Schmidt, K. (2017). Maternal perinatal anxiety: a review of prevalence and correlates. Clin. Psychol. 21, 4-19. doi: 10.1111/cp. 12058

Lecrubier, Y., Sheehan, D., Weiller, E., Amorim, P., Bonora, I., Harnett Sheehan, K., et al. (1997). The mini international neuropsychiatric interview (MINI). a short diagnostic structured interview: reliability and validity according to the CIDI. Eur. Psychiatry 12, 224-231. doi: 10.1016/S0924-9338(97)83296-8

Marcos-Nájera, R., Le, H.-N., Rodríguez-Muñoz, M. F., Olivares Crespo, M. E., and Izquierdo Mendez, N. (2018). The structure of the patient health questionnaire9 in pregnant women in Spain. Midwifery 62, 36-41. doi: 10.1016/j.midw.2018. 03.011

Mauri, M., Oppo, A., Montagnani, M. S., Borri, C., Banti, S., Camilleri, V., et al. (2010). Beyond "postpartum depressions": specific anxiety diagnoses during pregnancy predict different outcomes. J. Affect. Disord. 127, 177-184. doi: 10. 1016/j.jad.2010.05.015

Mazzotti, E., Fassone, G., Picardi, A., Sagoni, E., Ramieri, L., Lega, I., et al. (2003). The patient health questionnaire (PHQ) for the screening of psychiatric disorders: a validation study versus the structured clinical interview for DSM-IV axis I (SCID-I). Ital. J. Psychopathol. 9, 235-242.

Meades, R., and Ayers, S. (2011). Anxiety measures validated in perinatal populations: a systematic review. J. Affect. Disord. 133, 1-15. doi: 10.1016/j.jad. 2010.10.009

Melville, J. L., Gavin, A., Guo, Y., Fan, M.-Y., and Katon, W. J. (2010). Depressive disorders during pregnancy. Obstet. Gynecol. 116, 1064-1070. doi: 10.1097/ AOG.0b013e3181f60b0a

Meneghetti, A. (2007). System and Personality. Rome: Ontopsicologia Editrice.

Meneghetti, A. (2011). Project human being. Rome: Ontopsicologia Editrice.

Milgrom, J. (2003). Depressione Postnatale. Ricerca, Prevenzione e Strategie di Intervento Psicologico. Trento: Erikson.

Milgrom, J., Ericksen, J., McCarthy, R., and Gemmill, A. W. (2006). Stressful impact of depression on early mother-infant relations. Stress Health 22, 229-238. doi: $10.1002 /$ smi.1101

Milgrom, J., and Gemmil, A. W. (2015). Identifying Perinatal Depression and Anxiety: Evidence-Based Practice in Screening, Psychosocial Assessment and Management. Malden, MA: John Wiley \& Sons.

Milgrom, J., Gemmill, A. W., Ericksen, J., Burrows, G., Buist, A., and Reece, J. (2015a). Treatment of postnatal depression with cognitive behavioural therapy, 
sertraline and combination therapy: a randomised controlled trial. Aust. New Zeal. J. Psychiatry 49, 236-245. doi: 10.1177/0004867414565474

Milgrom, J., Holt, C., Holt, C. J., Ross, J., Ericksen, J., and Gemmill, A. W. (2015b). Feasibility study and pilot randomised trial of an antenatal depression treatment with infant follow-up. Arch. Womens. Ment. Health 18, 717-730. doi: 10.1007/s00737-015-0512-5

Milgrom, J., Martin, P. R., and Negri, L. M. (1999). Treating Postnatal Depression: A Psychological Approach for Health Care Practitioners. Chichester: Wiley.

Milgrom, J., Schembri, C., Ericksen, J., Ross, J., and Gemmill, A. W. (2011). Towards parenthood: an antenatal intervention to reduce depression, anxiety and parenting difficulties. J. Affect. Disord. 130, 385-394. doi: 10.1016/j.jad. 2010.10.045

Mirabella, F., Michielin, P., Piacentini, D., Veltro, F., Barbano, G., Cattaneo, M., et al. (2016). Efficacia di un intervento psicologico rivolto a donne positive allo screening per depressione post partum. Riv. Psichiatr. 51, 260-269. doi: $10.1708 / 2596.26728$

Misri, S., and Swift, E. (2015). Generalized anxiety disorder and major depressive disorder in pregnant and postpartum women: maternal quality of life and treatment outcomes. J. Obstet. Gynaecol. Canada 37, 798-803. doi: 10.1016/ S1701-2163(15)30150-X

Mortazavi, F., Mousavi, S. A., Chaman, R., and Khosravi, A. (2014). Maternal quality of life during the transition to motherhood. Iran. Red Crescent Med. J. 16:e8443. doi: 10.5812/ircmj.8443

Moura, M. R. S., Araújo, C. G. A., Prado, M. M., Paro, H. B. M. S., Pinto, R. M. C., Abdallah, V. O. S., et al. (2017). Factors associated with the quality of life of mothers of preterm infants with very low birth weight: a 3-year follow-up study. Qual. Life Res. 26, 1349-1360. doi: 10.1007/s11136-0161456-6

Nascimento, S., Surita, F., Parpinelli, M., Siani, S., Pinto, E., and Silva, J. (2011). The effect of an antenatal physical exercise programme on maternal/perinatal outcomes and quality of life in overweight and obese pregnant women: a randomised clinical trial. BJOG Int. J. Obstet. Gynaecol. 118, 1455-1463. doi: 10.1111/j.1471-0528.2011.03084.x

O'Connor, E., Rossom, R. C., Henninger, M., Groom, H. C., and Burda, B. U. (2016). Primary care screening for and treatment of depression in pregnant and postpartum women. JAMA 315, 388-406. doi: 10.1001/jama.2015.18948

Okagbue, H. I., Adamu, P. I., Bishop, S. A., Oguntunde, P. E., Opanuga, A. A., and Akhmetshin, E. M. (2017). Systematic review of prevalence of antepartum depression during the trimesters of pregnancy. Open Access Maced. J. Med. Sci. 7, 1555-1560. doi: 10.3889/oamjms.2019.270

Palumbo, G., Mirabella, F., Barbano, G., Cattaneo, M., and Gigantesco, A. (2018). "Dalla ricerca alla pratica: prevenzione e intervento precoce per il rischio di depressione post partum," in Il Futuro dei Primi Mille Giorni di Vita, A. Imbasciati and L. Cena (Milan: FrancoAngeli).

Palumbo, G., Mirabella, F., Cascavilla, I., Del Re, D., Romano, G., and Gigantesco, A. (2016). Prevenzione e Intervento Precoce per il Rischio di Depressione Post Partum. (Rapporti ISTISAN 16/31). Rome: Istituto Superiore di Sanità.

Palumbo, G., Mirabella, F., Imbasciati, A., Cena, L., Barbano, G., Cattaneo, M., et al. (2017). Prevenzione della sofferenza psichica perinatale. Not. dell'Istituto Super. Sanità 30, 3-7.

Parfitt, Y., and Ayers, S. (2014). Transition to parenthood and mental health in first-time parents. Infant Ment. Health J. 35, 263-273. doi: 10.1002/imhj.21443

Pedrabissi, L., and Santinello, M. (1992). STAI: State-Trait Anxiety InventoryForma Y-Manual. Firenze: Organizzazioni Speciali.

Reck, C., Struben, K., Backenstrass, M., Stefenelli, U., Reinig, K., Fuchs, T., et al. (2008). Prevalence, onset and comorbidity of postpartum anxiety and depressive disorders. Acta Psychiatr. Scand. 118, 459-468. doi: 10.1111/j.16000447.2008.01264.x

Riva Crugnola, C., Ierardi, E., Ferro, V., Gallucci, M., Parodi, C., and Astengo, M. (2016). Mother-infant emotion regulation at three months: the role of maternal anxiety, depression and parenting stress. Psychopathology 49, 285-294. doi: $10.1159 / 000446811$

Robinson, G. E., and Stewart, D. E. (2001). "Postpartum disorders," in Psychological Aspects of Women's Health Care, 2nd Edn, eds N. L. Stotland and D. E. Stewart (Washington. DC: American Psychiatric Press), 117-139.

Rossi, A., Alberio, R., Porta, A., Sandri, M., Tansella, M., and Amaddeo, F. (2004). The reliability of the mini-international neuropsychiatric interview - Italian version. J. Clin. Psychopharmacol. 24, 561-563. doi: 10.1097/01.jcp.0000139758. 03834.ad

Santos, I. S., Tavares, B. F., Munhoz, T. N., Manzolli, P., de Ávila, G. B., Jannke, E., et al. (2016). Patient health questionnaire-9 versus Edinburgh postnatal depression scale in screening for major depressive episodes: a cross-sectional population-based study. BMC Res. Notes 9:453. doi: 10.1186/s13104-0162364-0

Sheehan, D. V., Lecrubier, Y., Sheehan, K. H., Amorim, P., Janavs, J., Weiller, E., et al. (1998). The mini-international neuropsychiatric interview (M.I.N.I.): the development and validation of a structured diagnostic psychiatric interview for DSM-IV and ICD-10. J. Clin. Psychiatry 59, 22-33.

Shonkoff, M., Duncan, G. J., and Yoshikawa, H. (2019). Maternal Depression can Undermine the Development of Young Children. Centre on the Developing Child. Cambridge, MA: Harvard University.

Sidebottom, A. C., Harrison, P. A., Godecker, A., and Kim, H. (2012). Validation of the patient health questionnaire (PHQ)-9 for prenatal depression screening. Arch. Womens Ment. Health 15, 367-374. doi: 10.1007/s00737-0120295-x

Sipsma, H. L., Callands, T., Desrosiers, A., Magriples, U., Jones, K., Albritton, T., et al. (2016). Exploring trajectories and predictors of depressive symptoms among young couples during their transition to parenthood. Matern. Child Health J. 20, 2372-2381. doi: 10.1007/s10995-016-2064-3

Smith-Nielsen, J., Matthey, S., Lange, T., and Væver, M. S. (2018). Validation of the edinburgh postnatal depression scale against both DSM-5 and ICD-10 diagnostic criteria for depression. BMC Psychiatry 18:393. doi: 10.1186/s12888018-1965-7

Spielberger, C. D. (1989). State-Trait Anxiety Inventory: Bibliography, 2nd Edn. Palo Alto, CA: Consulting Psychologists Press.

Spielberger, C. D., Gorsuch, R. L., Luschene, R., Vagg, P. R., and Jacobs, G. A. (1983). Manual for the State-Trait Anxiety Inventory STAI (Form Y)(" SelfEvaluation Questionnaire"). Palo Alto, CA: Consulting Psychologists Press.

Spitzer, R. L., Williams, J. B. W., and Kroeneke, K. (2014). Test review: patient health questionnaire-9 (PHQ-9). Rehabil. Couns. Bull. 57, 246-248. doi: 10. $1177 / 0034355213515305$

Stefana, A., and Lavelli, M. (2017). Parental engagement and early interactions with preterm infants during the stay in the neonatal intensive care unit: protocol of a mixed-method and longitudinal study. BMJ Open 7:e013824. doi: 10.1136/ bmjopen-2016-013824

Suri, R., Lin, A. S., Cohen, L. S., and Altshuler, L. L. (2014). Acute and longterm behavioral outcome of infants and children exposed in utero to either maternal depression or antidepressants. J. Clin. Psychiatry 75, e1142-e1152. doi: 10.4088/JCP.13r08926

Thase, M. E. (2016). Recommendations for screening for depression in adults. JAMA 315, 349-350. doi: 10.1001/jama.2015.18406

The Whoqol Group. (1998). Development of the world health organization WHOQOL-BRIEF quality of life assessment. Psychol. Med. 28, 551-558. doi: $10.1017 / \mathrm{s} 0033291798006667$

Ukatu, N., Clare, C. A., and Brulja, M. (2018). Postpartum depression screening tools: a review. Psychosomatics 59, 211-219. doi: 10.1016/j.psym.2017. 11.005

Underwood, L., Waldie, K., D'Souza, S., Peterson, E. R., and Morton, S. (2016). A review of longitudinal studies on antenatal and postnatal depression. Arch. Womens Ment. Health 19, 711-720. doi: 10.1007/s00737-0160629-1

van der Waerden, J., Galéra, C., Larroque, B., Saurel-Cubizolles, M.-J., SutterDallay, A.-L., and Melchior, M. (2015). Maternal depression trajectories and children's behavior at age 5 years. J. Pediatr. 166, 1440.e-1448.e. doi: 10.1016/ j.jpeds.2015.03.002

Vizzini, L., Popovic, M., Zugna, D., Vitiello, B., Trevisan, M., Pizzi, C., et al. (2018). Maternal anxiety, depression and sleep disorders before and during pregnancy, and preschool ADHD symptoms in the NINFEA birth cohort study. Epidemiol. Psychiatr. Sci. 28, 521-531. doi: 10.1017/S204579601800 0185

Webster, J., Nicholas, C., Velacott, C., Cridland, N., and Fawcett, L. (2010). Validation of the WHOQOL-BREF among women following childbirth. Aust. New Zeal. J. Obstet. Gynaecol. 50, 132-137. doi: 10.1111/j.1479-828X.2009. 01131.x 
World Health Organization (1992). The ICD-10 Classification of Mental and Behavioural Disorders: Clinical Descriptions and Diagnostic Guidelines. Geneva: World Health Organization.

Yawn, B. P., Pace, W., Wollan, P. C., Bertram, S., Kurland, M., Graham, D., et al. (2009). Concordance of edinburgh postnatal depression scale (EPDS) and patient health questionnaire (PHQ-9) to assess increased risk of depression among postpartum women. J. Am. Board Fam. Med. 22, 483-491. doi: 10.3122/ jabfm.2009.05.080155

Yeaton-Massey, A., and Herrero, T. (2019). Recognizing maternal mental health disorders. Curr. Opin. Obstet. Gynecol. 31, 116-119. doi: 10.1097/GCO. 0000000000000524

Zhong, Q., Gelaye, B., Rondon, M., Sánchez, S. E., García, P. J., Sánchez, E., et al. (2014). Comparative performance of patient health questionnaire-9 and edinburgh postnatal depression scale for screening antepartum depression. J. Affect. Disord. 162, 1-7. doi: 10.1016/j.jad.2014. 03.028

Conflict of Interest: The authors declare that the research was conducted in the absence of any commercial or financial relationships that could be construed as a potential conflict of interest.

Copyright () 2020 Cena, Palumbo, Mirabella, Gigantesco, Stefana, Trainini, Tralli and Imbasciati. This is an open-access article distributed under the terms of the Creative Commons Attribution License (CC BY). The use, distribution or reproduction in other forums is permitted, provided the original author(s) and the copyright owner(s) are credited and that the original publication in this journal is cited, in accordance with accepted academic practice. No use, distribution or reproduction is permitted which does not comply with these terms. 